\title{
An Autofocus Approach for Residual Motion Errors With Application to Airborne Repeat-Pass SAR Interferometry
}

\author{
Karlus A. Câmara de Macedo, Member, IEEE, Rolf Scheiber, and Alberto Moreira, Fellow, IEEE
}

\begin{abstract}
Airborne repeat-pass SAR systems are very sensible to subwavelength deviations from the reference track. To enable repeat-pass interferometry, a high-precision navigation system is needed. Due to the limit of accuracy of such systems, deviations in the order of centimeters remain between the real track and the processed one, causing mainly undesirable phase undulations and misregistration in the interferograms, referred to as residual motion errors. Up to now, only interferometric approaches, as multisquint, are used to compensate for such residual errors. In this paper, we present for the first time the use of the autofocus technique for residual motion errors in the repeat-pass interferometric context. A very robust autofocus technique has to be used to cope with the demands of the repeat-pass applications. We propose a new robust autofocus algorithm based on the weighted least squares phase estimation and the phase curvature autofocus (PCA) extended to the range-dependent case. We call this new algorithm weighted PCA. Different from multisquint, the autofocus approach has the advantage of being able to estimate motion deviations independently, leading to better focused data and correct impulse-response positioning. As a consequence, better coherence and interferometric-phase accuracy are achieved. Repeat-pass interferometry based only on image processing gains in robustness and reliability, since its performance does not deteriorate with time decorrelation and no assumptions need to be made on the interferometric phase. Repeat-pass data of the E-SAR system of the German Aerospace Center (DLR) are used to demonstrate the performance of the proposed approach.
\end{abstract}

Index Terms-Airborne, autofocus, differential syntheticaperture-radar interferometry (D-InSAR), estimation, residual motion error.

\section{INTRODUCTION}

D EVIATIONS of the synthetic-aperture-radar (SAR) antenna from the reference track cause mismatch between the echo signal and the reference chirp used for SAR focusing. This mismatch gives origin to phase errors in the signal history. These errors cause broadening of the SAR impulse-response function (IRF) (defocus) and target-position errors in range and azimuth directions [1]-[3]. Particularly, airborne systems are affected due to the flight instabilities. Such phase errors can be cancelled by measuring, through a navigation system, the

Manuscript received June 30, 2007; revised November 23, 2007. Current version published October 1, 2008.

K. A. C. de Macedo was with Microwaves and Radar Institute, German Aerospace Center (DLR), 82230 Oberpfaffenhofen, Germany. He is now with the Orbisat Remote Sensing, 13025-320 São José dos Campos-SP, Brazil (e-mail: karlus.macedo@orbisat.com.br).

R. Scheiber and A. Moreira are with Microwaves and Radar Institute, German Aerospace Center (DLR), 82230 Oberpfaffenhofen, Germany (e-mail: rolf.scheiber@dlr.de; alberto.moreira@dlr.de).

Digital Object Identifier 10.1109/TGRS.2008.924004 deviations and subsequent removal with Motion Compensation (MoComp). These measurements have to be performed at subwavelength scale from pulse to pulse to obtain high-resolution SAR images. This puts an extreme burden on the navigation system. Autofocus techniques are used to estimate phase errors beyond the capability of the navigation system [4]-[8]. In addition, if no navigation data are available, autofocus can be used to achieve an equivalent performance to lower precision motion-measurement instrumentation.

With the use of up-to-date navigation instrumentation (D-GPS/INS), with precision in the order of centimeters, in SAR systems like E-SAR, [9], [10], AIRSAR [11], and Orbisar [12], there is practically no defocusing on highresolution SAR data and no need to use autofocus. For very high resolution data, broadening of the SAR IRF still affects the resolution and requires basic autofocus [13].

Even when there is no relevant defocus in the SAR image, we still have motion errors that cause mainly misregistration and undesirable phase undulations in the interferograms. These motion errors shall be referred to as residual motion errors. For single-pass interferometry, most of the residual motion errors cancel out due to the signal beating of the two interferometric channels, which have practically the same deviations. Differently, for repeat-pass interferometry, the channels have uncorrelated motion errors causing undesired phase artifacts (up to one fringe in L-band and several fringes in X-band) in the final interferogram for motion errors in the order of centimeters [14]-[18].

To cope with the recent demands of airborne repeat-pass interferometry and its applications, such as differential SAR interferometry (D-InSAR) which measures terrain movements at millimeter scale, it is necessary to estimate phase errors with accuracy under $0.25 \mathrm{rad}$. This requirement motivated the development of algorithms to estimate phase errors from interferometric images, such as multisquint [15], [16]. It is based on the estimation of the misregistration values [19]. The multisquint approach has shown to be robust with the improvement of coherence and decrease of phase errors in repeatpass interferograms through its estimations. The multisquint, however, is only able to estimate the baseline variations from coregistered images. An autofocus approach has the advantage of being able to estimate phase errors independently for each image, not requiring any interferometric process or assumption on the interferometric phase.

Up to now, autofocus approaches with algorithms such as Map Drift [4] or Phase-Gradient Autofocus (PGA) [5], [7], [8] have not been suggested for residual-motion estimation in a 
repeat-pass interferometric context. The reason for this is based on the fact that typical autofocus techniques lead to estimation errors on the order of $0.5-1.0 \mathrm{rad}$, which is above the desired accuracy for repeat-pass interferometry. Nevertheless, this estimation error can be decreased using recent improvements in the PGA algorithms as, for example, the quality PGA (QPGA) algorithm or the weighted least squares (WLS) phase estimation presented in [20] and [21], respectively. The second reason is that current airborne systems, that are equipped with up-todate high-precision navigation instrumentation and are able to perform repeat-pass interferometry, operate in stripmap mode. Since PGA was first thought for spotlight mode [22], an adaptation of the PGA algorithm, such as phase curvature autofocus (PCA), to work in stripmap mode was suggested [23]. But, due to the nonrange dependence and double-differentiation nature of this approach, there is an increase in the estimation error, which makes it not reliable when high precision is desired.

This paper proposes a new robust autofocus algorithm to be used in combination with a modern navigation system and capable of performing phase estimations under 0.25-rad accuracy in order to enable repeat-pass interferometric applications. It is based on the WLS phase estimation and on PCA extended to the range-dependent case. We call this new algorithm weighted PCA (WPCA). An autofocus approach has the advantage of not depending on the interferometric coherence, because the residual errors can be independently estimated for each SAR image. It opens the possibility to perform robust airborne D-InSAR for low coherence and long-term interferograms at any frequency band. Additionally, the interferometric-phase changes related to the along-track terrain displacements are not mixed up with the motion estimation, as in the case with multisquint where assumptions on the interferometric phase have to be made [24].

This paper is organized as follows. Section II is dedicated to a summary of robust phase-estimation algorithms such as PGA, PCA, and multisquint. Section III presents the new autofocus technique for residual motion errors (WPCA). In Section IV, repeat-pass data at L-band of the E-SAR system of the German Aerospace Center (DLR) are used to demonstrate the performance of the proposed WPCA algorithm. In Section V, we address the conclusions.

\section{Phase-Error-Estimation Algorithms}

\section{A. $P G A$}

The PGA is an algorithm to estimate the phase errors in SAR images from the SAR data [5], [7], [8]. It was developed to operate in spotlight mode. It has been widely used by the SAR community and was shown to be very robust on a variety of imagery and phase-error functions. PGA exploits the redundancy of the motion error along scatterers at different range bins. In spotlight mode, the deramped signal of a point target in range-compressed time domain can be modeled as

$$
g_{s}(t)=\left|g_{s}(t)\right| \exp \left(-j\left(\omega_{o} t+\nu_{o}+\phi_{e}(t)\right)\right)+\nu_{\eta}(t)
$$

where $\phi_{e}(t)$ is the phase of the signal associated with motion error, $\omega_{o}$ is the frequency of the deramped signal, $\nu_{o}$ is the phase-offset contribution of the strongest scatterer, and $\nu_{\eta}(t)$ is the contribution of all the other scatterers treated as clutter.
The PGA algorithm encompasses the selection of strong targets, the shift of the targets to the center of the image to remove the frequency of the scatterers, and the windowing of the targets to preserve the width of the dominant blur. After these steps, the signal $g_{s}(t)$ becomes

$$
g(t)=|g(t)| \exp \left[-j\left(\phi_{e}(t)+\nu_{o}\right)\right]+\nu_{\eta}(t) .
$$

In order to coherently combine several targets into the estimation process, PGA models the clutter $\nu_{\eta}(t)$ as uncorrelated noise and estimates phase gradients to handle targets with different phase offsets $\nu_{o}$ and to remove the integer ambiguity of the modulo $2 \pi$.

Then, the PGA algorithm integrates the estimated phase gradient to obtain the phase errors (except for an integral constant) and, finally, removes them from the SAR image implementing a motion-compensation technique. These steps are repeated iteratively, decreasing the window size, until the algorithm converges. The estimation kernel of the PGA algorithm is

$$
\hat{\dot{\phi}}_{e}(t)=\frac{\sum_{k=1}^{K} \operatorname{Im}\left[\dot{g}_{k}(t) g_{k}^{*}(t)\right]}{\sum_{k=1}^{K}\left|g_{k}(t)\right|^{2}}
$$

where $\dot{\phi}_{e}$ is the estimated phase-error gradient and $g_{k}(t)$ is the signal associated with the shifted and windowed target in the $k$ th range bin and $\dot{g}_{k}(t)$ is its derivative. The $\operatorname{Im}[\cdot]$ denotes the imaginary part of $[\cdot]$.

The PGA phase estimation implements the linear minimumvariance estimator as long as the statistics of the phase gradient remains the same. If the data are corrupted by white Gaussian noise (WGN), the PGA implements a linear minimum-variance unbiased estimator [5].

Modification of the estimation kernel or target-selection criteria have been proposed to optimize the estimation performance. In [7], a maximum-likelihood (ML) phase-gradientestimation kernel is developed and is given by

$$
\hat{\dot{\phi}}_{e}^{\mathrm{ML}}(l)=\arg \sum_{k=1}^{K}\left[g_{k, l}^{*} g_{k, l+1}\right]
$$

where $l$ is the azimuth bin index. Assuming that the data are corrupted by WGN, the ML algorithm converges faster then the original PGA kernel [(3)].

Target-selection criteria were also modified. Conventional PGA selects the brightest pixel on a range line as being a target with high signal-to-clutter ratio (SCR). This model works well for most cases, but it fails in areas consisting of strong clutter or strong distributed targets. The authors of [20] present the QPGA algorithm, in which the selection criteria is modified according to the targets' SCR. Additionally, strong targets at the same range line are used to optimize the estimation. QPGA shows a better estimation performance when compared to PGA, and a noniterative approach is possible.

\section{B. WLS for Autofocus}

The authors of [21] also exploit the targets that have high SCR. They derive a WLS phase estimator where the phase 
signal is weighted according to its SCR. This phase estimation is more robust than the ones implemented by PGA and ML, because it does not require the clutter variance to be the same for the whole image or of a certain model. The weighting optimizes the estimation in images made up of distributed targets with different phase statistics (nonstationary noise). The WLS is the minimum-variance estimator for any uncorrelated noise process [25]. The WLS phase estimation is given by

$$
\hat{\mathbf{P}}_{\mathbf{e}}=\left(\mathbf{A}^{\mathrm{T}} \mathbf{W A}\right)^{-1} \mathbf{A}^{\mathrm{T}} \mathbf{W P}
$$

with covariance of the estimation

$$
\mathbf{C}=\left(\mathbf{A}^{\mathrm{T}} \mathbf{W A}\right)^{-1}
$$

where $\mathbf{P}$ is a vector with the observed phase signals (first or second phase derivatives or unwrapped phase) of several range bins, $\mathbf{W}$ is the diagonal matrix with the weight values, $\mathbf{A}$ is the model of the phase signal, and $\hat{\mathbf{P}}_{e}$ is the estimated parameter vector with the phase errors associated with the motion deviations.

In [21], the WLS kernel for autofocus is derived for $\mathbf{A}$ equal to one (no range dependence is considered), and the observed signal is the unwrapped phase $\phi_{k}(t)$. Thus, the estimated phase error $\hat{\phi}_{e}^{W}$ is given by

$$
\hat{\phi}_{e}^{W}(t)=\sum_{k=1}^{K} \frac{w_{k} \phi_{k}(t)}{\sum_{j=1}^{K} w_{j}}
$$

where $w_{k, j}$ are the weights in the $k=j=0,1, \ldots, K$ th range bin.

The absolute phases $\phi_{k}(t)$ are obtained using the localphase-unwrapping technique [21]. As a result, it is not necessary to compute phase gradients avoiding the increase of noise in the estimation process.

The weight $w$ for a range bin is the inverse of the phase variance $\sigma_{\nu}^{2}$ of the signal in that bin. In [21], the relationship between the phase variance and the second-order statistics of the amplitude (contrast) of the range-decompressed signal $g(t)$ is shown. Thus, it follows that

$$
w=1 / \sigma_{\nu}^{2} \approx 1 /\left((1 / 2) R+(5 / 24) R^{2}\right)
$$

where $R$ is the inverse of the SCR and is given by

$$
R \approx \frac{1}{d}\left(4\left(2 c^{2}-d\right)-4 c \sqrt{4 c^{2}-3 d}\right)
$$

with

$$
\begin{aligned}
& c=E[|g(t)|] \\
& d=E\left[|g(t)|^{2}\right]
\end{aligned}
$$

where $E[\cdot]$ is the expectation operator.

The WLS autofocus has been shown to be superior to the PGA and ML algorithms, improving performance in distributed target areas [21].

\section{Autofocus for Stripmap Mode}

The PGA framework cannot be directly applied to stripmap SAR images. In [23], the modifications of this framework are presented to enable phase-error estimation in the stripmap case. The new framework begins with the windowing of the selected targets on the focused data. No shift of the targets is performed, since in stripmap, no movable antenna is steered to illuminate a single spot. Then, the windowed focused signal $f(t)$ is convolved with the reference SAR chirp $s_{r}(t)$. This step corresponds to a decompression. This will bring the windowed signal to the range-compressed domain (not compressed in azimuth). The azimuth-decompressed signal is then multiplied by the conjugate complex of the reference chirp (deramping). The result is equivalent to the range-compressed spotlight signal. This procedure can be described mathematically as follows:

$$
g(t)=\mathcal{C}\{f(t)\}=\mathcal{F}\left\{\mathcal{F}\{f(t)\} \mathcal{F}\left\{s_{r}(t)\right\}\right\}^{-1} s_{r}^{*}(t)
$$

where $\mathcal{C}\{\cdot\}$ denotes the transformation from a stripmap signal to a spotlight signal with $\mathcal{F}\{\cdot\}$ as the Fourier transform.

The windowed range-decompressed signals are aligned according to their azimuth time of acquisition in order to combine several phase estimates. Due to the fact that targets are imaged with different or partially overlapped apertures, the target positions are inaccurately estimated, leading to individual unknown linear components. In order to coherently combine several targets into the estimation process, the autofocus for stripmap SAR data estimates the phase double differences, i.e., phase accelerations. Thus, a double integration is needed to obtain the phase errors. As a consequence, only the curvature of the phase error can be recovered. For that reason, autofocus for stripmap mode is often referred to as PCA algorithm. Due to the double differentiation of the signals, there is an inherent increase of the noise in the estimation process as compared to PGA. This makes the use of PCA less robust. In practice, PCA has not been widely used.

The work in [26], the phase-weighted-estimation PGA (PWE-PGA) presents an estimation kernel for stripmap data. It computes phase gradients $\dot{\phi}$ instead of double derivatives by converting blocks of the SAR stripmap image into spotlight representation. Additionally, the PWE-PGA introduces a WLS kernel for the phase-gradient estimation with a range-dependent model. The weights come from the ML formulation. The rangedependent model is fundamental for low-altitude SAR systems like the E-SAR. In such systems, the changes in the look angle cannot be neglected. For a SAR sensor with 3000-m altitude above ground, the deviation of the track of $0.05 \mathrm{~m}$ in horizontal and vertical directions causes a phase error that varies $2.0 \mathrm{rad}$ from near $\left(30^{\circ}\right)$ to far range $\left(60^{\circ}\right)$ at L-band. Therefore, autofocus with a nonrange-dependent model can cause an error up to $1.0 \mathrm{rad}$ in the estimated phase. The range-dependent model decomposes the phase error $\phi_{e}$ into a vertical and a horizontal component that are directly related to the vertical and horizontal motion deviations. The WLS formulation of the PWE-PGA is given by

$$
\begin{aligned}
\dot{\phi}_{k} & =\arg \left[g_{k, l}^{*} g_{k, l+1}\right] \\
m_{k} & =\left|g_{k, l}^{*} g_{k, l+1}\right|
\end{aligned}
$$




$$
\begin{aligned}
\mathbf{A} & =\left[\begin{array}{cc} 
\pm \sin \theta_{1} & \cos \theta_{1} \\
\vdots & \vdots \\
\pm \sin \theta_{K} & \cos \theta_{K}
\end{array}\right] \\
\mathbf{W} & =\operatorname{diag}\left[m_{1}, \ldots, m_{K}\right], \quad k=1,2,3, \ldots, K \\
\mathbf{P} & =\left[\begin{array}{c}
\dot{\phi}_{1} \\
\vdots \\
\dot{\phi}_{K}
\end{array}\right], \quad k=1,2,3, \ldots, K \\
\hat{\mathbf{D}}_{\mathbf{y z}} & =\left[\begin{array}{c}
\hat{\dot{D}}_{y} \\
\hat{\dot{D}}_{z}
\end{array}\right]=\frac{\lambda}{4 \pi} \hat{\mathbf{P}}_{e}=\frac{\lambda}{4 \pi}\left[\begin{array}{c}
\hat{\phi}_{y} \\
\hat{\dot{\phi}}_{z}
\end{array}\right]
\end{aligned}
$$

where $\dot{\phi}_{k}$ is a phase-gradient observation at the $k$ th range bin, $m_{k}$ is the corresponding weight value, $\theta_{k}$ is the look angle for a certain range bin of the range-dependent model matrix $\mathbf{A}, \hat{\dot{\phi}}_{y}$ and $\hat{\dot{\phi}}_{z}$ are the horizontal and vertical components of the phaseerror derivatives, respectively, and $\hat{\dot{D}}_{y}$ and $\hat{\dot{D}}_{z}$ are the corresponding horizontal and vertical components of the motion deviations from the reference track, respectively. The \pm sign depends on the side-looking geometry. Positive signal for left and negative for right-looking antennas.

The synthetic aperture sonar community has recently been working on reliable autofocus methods for sonar systems, which operate in stripmap mode. Due to the not wide use of the PCA algorithm, only one kernel (nonrange-dependent), for the ML estimator, has been derived and used for phase-doubledifference estimation [27]

$$
\hat{\ddot{\phi}}_{e}^{\mathrm{ML}}(l)=\arg \sum_{k=1}^{K}\left[g_{k, l-1}\left(g_{k, l}^{*}\right)^{2} g_{k, l+1}\right] \text {. }
$$

Some estimation difficulties have been reported in [27] and [28] when using PCA due to the double differentiation. As a result, the phase-matching autofocus (PMA) and the stripmap PGA (SPGA) have been proposed in [27], [29], and [30]. They suggest a PCA/PGA algorithm hybrid solution. These algorithms use the fact that a linear component causes a shift in the signal frequency domain. The Doppler centroid estimation [31] is used to retrieve the unknown linear component, and as a consequence, phase gradients can be used to estimate phase errors in stripmap images.

\section{Multisquint Approach}

The multisquint is an approach that was developed in order to compensate for residual motion errors that still persist in the interferometric data even after the use of high-precision navigation systems for MoComp. The main motivation is the use of SAR data for repeat-pass mode applications like D-InSAR.

Multisquint relies on an interferometric framework in order to obtain very robust and accurate residual baseline estimation. Multisquint is based on azimuth-misregistration-values estimation using spectral diversity [19]. It works by splitting the azimuth bandwidth of two SAR complex images $S_{1}, S_{2}$ into two sublooks $A$ and $B$ (or more) to form interferograms and, then, a differential interferogram $\Psi_{\text {diff, sub. Assuming that }}$ most of the phase of the differential interferogram are coming from the baseline errors, $\Psi_{\text {diff,sub }}$ becomes proportional to the derivative of the residual baseline deviations $\dot{\Psi}_{\Delta \text { base }}$ according to [15], [16], and [32]. It follows that an estimate for the derivative of the residual baseline is

$$
\dot{\psi}_{\Delta \text { base }, k}=\frac{\psi_{\text {diff }, \text { sub }}}{\Delta x}
$$

with

$$
\begin{aligned}
\Delta x & =r\left(\tan \left(\theta_{\mathrm{sqc}}^{A}\right)-\tan \left(\theta_{\mathrm{sqc}}^{B}\right)\right) \\
\psi_{\text {diff }, \text { sub }} & =\arg \left[s_{1}^{A} s_{2}^{A *}\left(s_{1}^{B} s_{2}^{B *}\right)^{*}\right]
\end{aligned}
$$

where $r$ is the target-to-sensor range and $\theta_{\text {sqc }}$ is the squint angle related to the center frequency of the sublooks.

In recent multisquint refinements [33], the interferometricphase-gradient kernel is also a WLS estimator with the range-dependent model of (15). The weights come from the interferometric coherence.

\section{Proposed Autofocus Algorithm for Residual MOTION ERRORS}

\section{A. WPCA Kernel}

We present here an autofocus approach for residual motion errors, i.e., estimation and compensation of motion deviations that are causing mainly misregistration and undesirable phase undulations. The motivation is the same as in the multisquint case, i.e., the use of airborne data for repeat-pass InSAR applications. As described in the previous section, multisquint is only able to estimate the residual baseline errors from coregistered images. As a result, the estimation performance decreases for large baselines and long-term interferograms. Additionally, the phase related to along-track terrain movement and baseline errors are mixed up together, leading to over or underestimations of the phase error [24]. To overcome those problems, autofocus could be a robust solution, since the images are processed independently, not requiring any interferometric processing. Thus, the estimation performance does not decrease with low-coherence data. Additionally, no assumptions need to be made on the interferometric-phase model. This would enable the generation of robust, reliable, and accurate SAR data for repeat-pass applications like D-InSAR. But, the current autofocus approaches are not able to deliver phase estimation to cope with millimeter motion error.

The PWE-PGA algorithm provides poor results for residualmotion-error estimation, due to the fact of not using double derivatives to coherently combine several estimates. Instead, it converts blocks of the stripmap SAR data into spotlight representation. This conversion introduces phase artifacts and discontinuities between blocks that can reach several centimeters, deteriorating residual-motion estimations. Additionally, the PWE-PGA performance is decreased, because it uses an ML formulation for the weights, which assumes WGN for the clutter. The only PCA kernel derived up to now [(19)] comes from the ML formulation and provides also poor results due to the increase of the power of the nonstationary noise after the double-differentiation process. Besides, it does not use a range-dependent geometry model. This model is necessary to enable phase estimations with accuracy better than $0.25 \mathrm{rad}$, as 
commented in Section II-C. The PMA and SPGA [29], [30] use Doppler centroid estimation to retrieve the unknown linear components; however, this estimation is not sufficiently reliable to handle subpixel shifts of each target, because it requires homogenous block of SAR data in order to properly estimate the Doppler centroid.

We propose an algorithm based on the PCA framework combined with a better target selection and an optimal, in the WLS sense, range-dependent phase kernel. We call this algorithm weighted phase curvature autofocus (WPCA). We start by deriving the estimation kernel. First, we take

$$
\ddot{g}(t)=\frac{\partial^{2}|g(t)| \exp [-j \phi(t)]}{\partial^{2} t}
$$

where $\phi(t)$ is the phase of the azimuth windowed signal $g(t)$. Since we already use the amplitude to compute the weights in the WLS formulation, we do not need to include the amplitude of the $g(t)$ signal to solve for $\ddot{\phi}(t)$, as in the case of the PGA and ML kernel. In fact, the amplitude of $g(t)$ does not affect the phase-gradient computation, and it is a good idea to force its amplitude to be equal to one to avoid increase of the noise. Solving the earlier equation for $\ddot{\phi}(t)$ and $|g(t)|=1$, we get

$$
\ddot{\phi}(t)_{k}=\left.\operatorname{Im}\left[\ddot{g}_{k}(t) g_{k}^{*}(t)+\dot{g}_{k}(t) \dot{g}_{k}^{*}(t)\right]\right|_{|g(t)|=1}
$$

where $\ddot{\phi}(t)_{k}$ is an observation or realization in the $k$ th range bin. The WLS formulation of the WPCA estimator uses the range-dependent model matrix $\mathbf{A}$ given in (15). The other matrices are

$$
\begin{aligned}
\mathbf{W} & =\operatorname{diag}\left[w_{1}, \ldots, w_{K}\right], \quad k=1,2,3, \ldots, K \\
\mathbf{P} & =\left[\begin{array}{c}
\ddot{\phi}_{1} \\
\vdots \\
\ddot{\phi}_{K}
\end{array}\right], \quad k=1,2,3, \ldots, K \\
\hat{\ddot{\mathbf{D}}}_{\mathbf{y z}} & =\left[\begin{array}{c}
\hat{\ddot{D}}_{y} \\
\hat{\ddot{D}}_{z}
\end{array}\right]=\frac{\lambda}{4 \pi} \hat{\mathbf{P}}_{\mathbf{e}}=\frac{\lambda}{4 \pi}\left[\begin{array}{c}
\hat{\ddot{\phi}}_{y} \\
\hat{\ddot{\phi}}_{z}
\end{array}\right]
\end{aligned}
$$

where the weight values $w_{k}$ are computed according to the SCR estimations (8) and (9), $\ddot{\phi}_{k}(t)$ is an observation of the double-derivative phase in the $k$ th range bin, $\hat{\ddot{\phi}}_{y}$ and $\hat{\ddot{\phi}}_{z}$ are the estimated double derivative of the horizontal and vertical components of the phase errors, respectively, and $\hat{\ddot{D}}_{y}$ and $\hat{\ddot{D}}_{z}$ are the corresponding horizontal and vertical acceleration of the motion deviations from the reference track, respectively.

\section{B. Filter to Avoid SCR Degradation}

Derivative operations work as a high-pass filter and, therefore, degrades the SCR due to the increase of the clutterphase undulations. To allow accurate derivative computation and avoid increase of white-noise clutter, we compute the phase double derivative using a numerical differentiation technique, different from the kernel in (19), which uses shift operations. Nevertheless, the main problem persists, since in practice, we also have nonwhite noise. In order to optimally use the WLS formulation in which the weights are computed according to the phase variance with (8), (9), (10), and (11), we have to keep the variance of the phase double derivatives in the same levels as before the differentiations. We do this by applying to $\hat{\ddot{\phi}}_{k}$ an autoregressive moving average (ARMA) to filter the clutter. This filter removes also high-frequency components of the residual motion error within the synthetic aperture. Thus, the filter length should be chosen according to the expected frequency of the residual motion error within the aperture and to the clutter power spectrum (for typical spectrum behavior of residual motion errors, please refer to [18]).

\section{WPCA Framework}

We implement a target preselection criteria that includes all pixels of the SAR images above a certain amplitude threshold $T_{\mathrm{A}}$. In a rectangular window of $\Omega_{\mathrm{rg}}$ and $\Omega_{\mathrm{az}}$ dimension in range and azimuth, respectively, we take the maximum-amplitude pixel. The next step is to window around this maximum pixel adaptively in azimuth with a window with dimension $\Omega$ corresponding to $-10 \mathrm{~dB}$ relative to the maximum. These targets are chosen as possible candidates. Then, each target candidate is converted into spotlight representation using $\mathcal{C}\{\cdot\}$ [(12)], i.e., it is decompressed and deramped. The reference chirp $s_{r}$ used for decompression of the data is

$$
s_{r}=\exp \left[\frac{j 4 \pi r_{0}}{\lambda}\left(1-\sqrt{1-\left(\frac{v_{0} t}{\sqrt{r_{0}^{2}+v_{0}^{2} t^{2}}}\right)^{2}}\right)\right]
$$

where $\lambda$ is the radar-signal wavelength, $v_{0}$ is the velocity of the SAR antenna, and $r_{0}$ is the range between the target and the SAR antenna.

After deramping, we obtain the signal $g(t)$, and (8), (9), (10), and (11) can be applied to compute the weights. If the spectrum of the focused data is filtered by an apodization function (for sidelobe suppression using Hamming window for example), we get a modulated amplitude signal $g_{H}(t)$, which disturbs the weight computations. To remove this modulation, we apply the inverse apodization before the deramping. Since this inverse apodization increases the noise, we apply it only when dealing with the weight computations. For the phase estimation, we use the signal with suppressed sidelobes. Therefore, the computed weights should be multiplied by the corresponding normalized apodization function.

Fig. 1 shows the block diagram for the weight computations and final target selection. Targets with weights less than a certain value $T_{w}$, or alternatively $\mathrm{SCR}$ less than $T_{\mathrm{SCR}}$, are discarded. The reason is to avoid wrong SCR estimations (false alarms), since [21] shows that SCR estimations are valid for values greater than $1 \mathrm{~dB}$.

We take now the selected targets with their corresponding weights and signals $g(t)$ and estimate the phase accelerations using (24). Each $\ddot{\phi}_{i}$ observation is then aligned according to its azimuth bin. To avoid undesired artifacts coming from the transient response of the windowed signal, the borders of each phase estimate are discarded. Effectively, half of the vector length is used. Note that each vector $\phi_{i}$ corresponds to a synthetic-aperture length.

The acceleration of the motion errors (horizontal and vertical) are then estimated using the WPCA, followed by double integration to determine the estimated motion deviations. Due to the unknown initial conditions $\phi_{y, z}(0)$ and $\dot{\phi}_{y, z}(0)$, we 


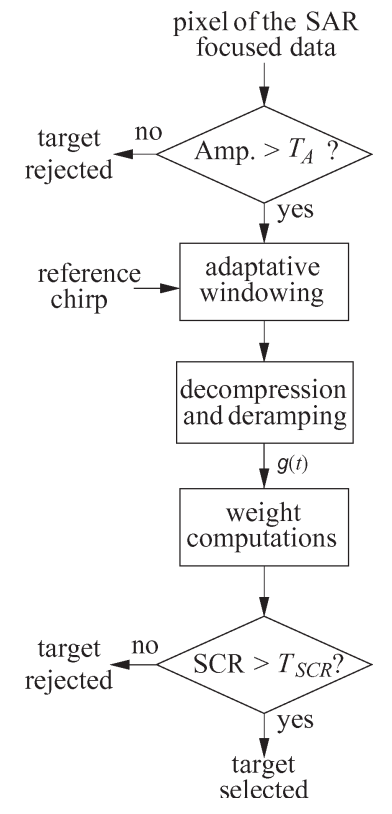

Fig. 1. Weight computation and target-selection flow diagram of the WPCA, where $T_{\mathrm{A}}$ and $T_{\mathrm{SCR}}$ are the thresholds for the amplitude and for the SCR values, respectively.

remove the global linear component and offset after the phase integration by subtracting a first-order polynomial. Finally, we use a topography- and aperture-dependent MoComp algorithm to remove the residual phase errors from the data [34], [35]. Fig. 2 shows the block diagram of the WPCA algorithm.

\section{Accuracy and Convergence}

Let us assume that the variance of the ARMA-filtered double-derivative phase estimates are approximately equal to the phase variance of the estimates given by (8), (9), (10), and (11). In this case, the covariance matrix of the WPCA phaseestimation process is

$$
\mathbf{C}_{\hat{\mathbf{\Phi}}_{\mathbf{y z}}} \approx\left(\mathbf{A}^{\mathrm{T}} \mathbf{W A}\right)^{-1}=\left[\begin{array}{ll}
C_{00} & C_{01} \\
C_{10} & C_{11}
\end{array}\right] .
$$

Considering the clutter as WGN, Fig. 3 shows the theoretical standard deviation of the phase estimations as a function of the SCR for different number of range bins, equally distributed around midrange $45^{\circ}$ look angle, for example, $\left[35^{\circ}, 55^{\circ}\right]$ $\left(\sqrt{C_{00}}=\sqrt{C_{11}}\right)$. The magnitude of the correlation coefficient between the estimated parameters are very strong, equal to 0.98 .

Furthermore, we assume that the number of estimates are large and diverse enough so that the bias of the acceleration estimations can be considered zero or constant along the azimuth bins. After the double integration, if the strip is long, a small constant bias may result in a quadratic curvature with amplitude greater than the desired accuracy. In this case, we force the mean value of the motion estimations along azimuth equal to zero. This procedure removes the constant bias but also a possible global quadratic component of the motion error. However, for long strips, it is very unlikely to have a relevant global quadratic term in the motion error.

After motion compensation, the WPCA is repeated iteratively, to refine the estimations, until the maximum of the norm

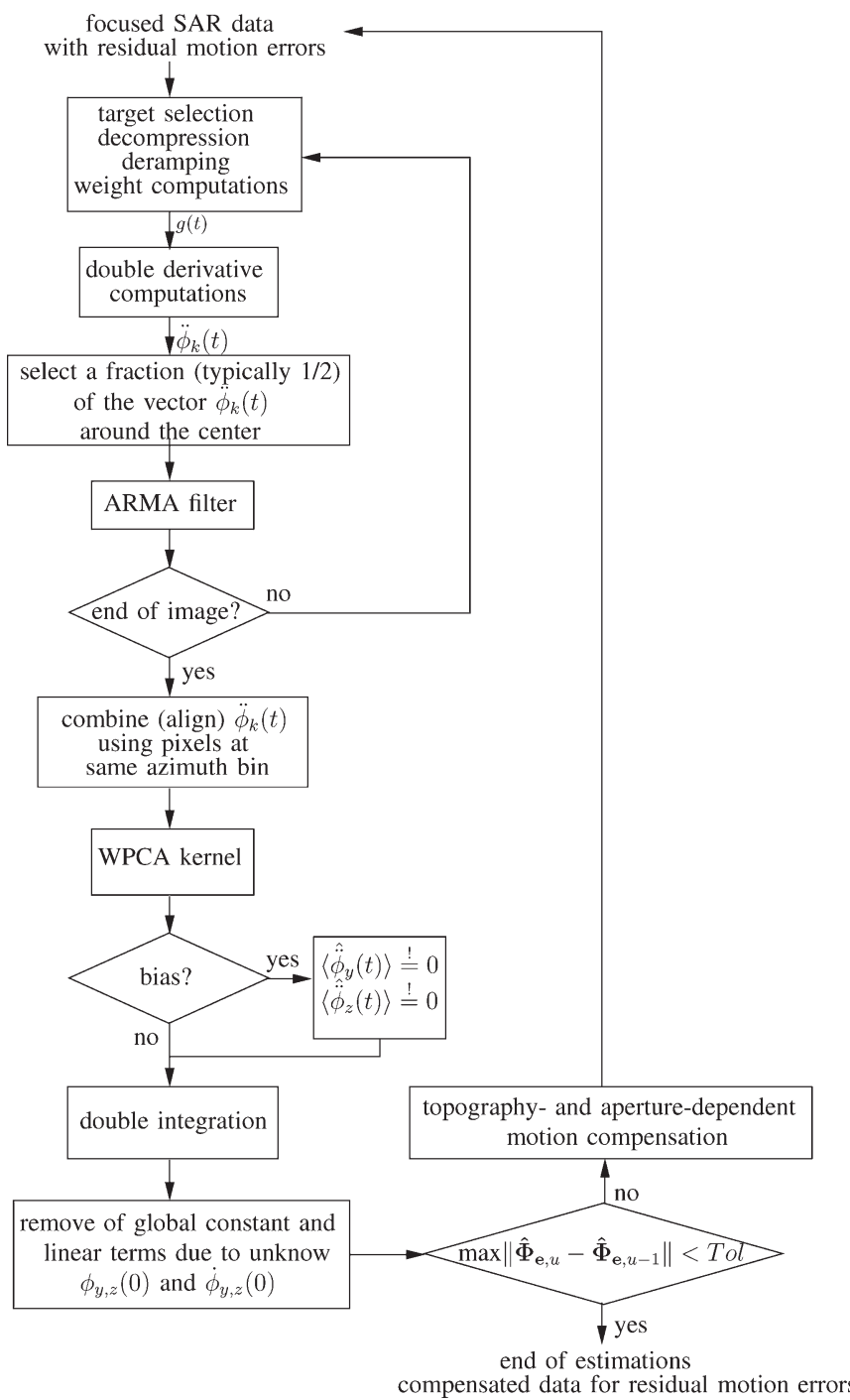

Fig. 2. WPCA-algorithm flow diagram, where $T o l$ is the tolerance of the stop criteria. The tolerance is chosen according to the theoretical estimation error.

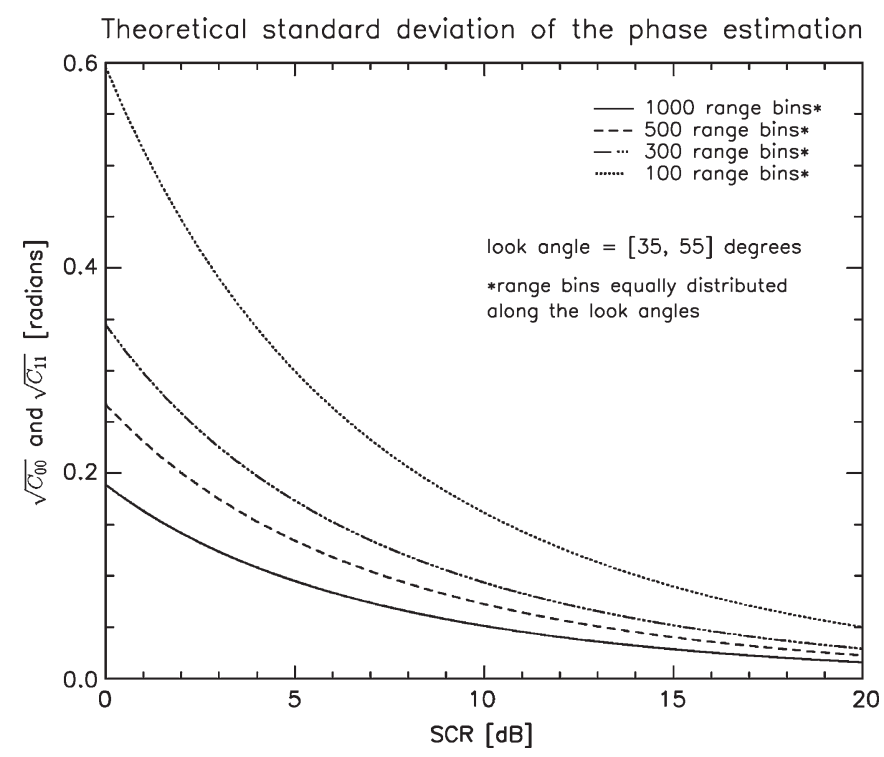

Fig. 3. Theoretical phase-estimation error as a function of the SCR for different number of range bins with look angles between $35^{\circ}$ and $55^{\circ}$. 
$\left\|\hat{\boldsymbol{\Phi}}_{\mathbf{e}, u}-\hat{\boldsymbol{\Phi}}_{\mathbf{e}, u-1}\right\|$, between successive iterations $u$, is equal or less than a tolerance value Tol (see Fig. 2). When this condition is satisfied, the WPCA achieved convergence. The tolerance can be defined as being greater than or equal to the theoretical phase accuracy times a value $\beta_{\phi}$. Thus, Tol is equal to

$$
\text { Tol }=\beta_{\phi} \sqrt{C_{00}+C_{11}} \text {. }
$$

The $\beta_{\phi}$ helps the algorithm to be robust against procedural errors that are not modeled by the assumptions.

\section{E. Estimation and Compensation of Global Terms}

Due to the double-integration nature of the process, there may exist remaining global terms of motion deviations, i.e., global constant and linear unknown terms, which can be estimated with interferometric data using the approach in [33]. This approach consists of modeling the interferometric phase minus the topographic phase from a digital elevation model (DEM), i.e., the residual interferogram $\psi^{\text {res }}$, as

$$
\psi_{k, l}^{\mathrm{res}}=\left(z_{0}+z_{1} l\right) \cos \theta_{k, l} \pm\left(y_{0}+y_{1} l\right) \sin \theta_{k, l}
$$

where $z_{0}, z_{1}, y_{0}$, and $y_{1}$ are the parameters of the model of the interferometric trend and $\psi_{k, l}^{\text {res }}$ is the residual interferometric phase at the $k$ th and $l$ th range and azimuth bin, respectively. The parameters of the model are estimated using WLS, where the weights come from the coherence of the interferometric data. The compensation is performed by subtracting the estimated trend from the interferometric data. If the bias was removed and the quadratic term of the motion error is relevant, then (31) can be extended to a second-order polynomial.

The global terms can be also removed with some few corner reflectors (CRs), which do not require modeling of the residual interferometric phase, and have the advantage of being very stable and independent of the geometrical and temporal baselines.

\section{RESULTS}

\section{A. WPCA Performance}

To demonstrate the performance and effectiveness of the WPCA algorithm for residual motion errors, we use real airborne repeat-pass data of the E-SAR system (DLR) acquired at L-band $(\mathrm{HH})$ in the same day with baseline length $B$ of $6.44 \mathrm{~m}$ (1.90- and 6.15-m horizontal and vertical baselines, respectively). This system uses an up-to-date high-precision navigation system (D-GPS/INS). For processing, we used the extended chirp scaling [9] followed by the precise-topographyand aperture-dependent MoComp [34]. Thus, the errors that persist in the images and interferograms are mainly residual motion errors due to the insufficient accuracy of the navigation system. The SAR images are of the Oberpfaffenhofen area, Germany, which is mostly agricultural with some man-made areas (buildings) and practically flat (see Fig. 4). The scene has $2.5 \mathrm{~km} \times 2.5 \mathrm{~km}$ (range times azimuth) dimension. The sensor altitude above ground is $3214 \mathrm{~m}$.

These scene was chosen to show the robustness of the WPCA algorithm even in vegetated areas. Autofocus algorithms have better performance in man-made areas where there are more pointlike targets. Nevertheless, since the WPCA does not model

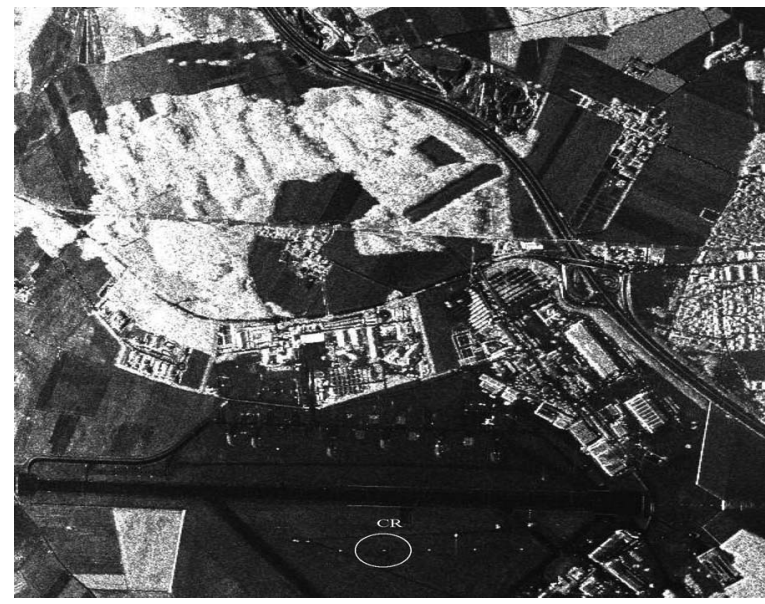

Fig. 4. Amplitude SAR Image (slave) at L-band used to demonstrate the WPCA algorithm. Range increases from left to right and azimuth from top to bottom.

the clutter but relies basically on the SCR, it is possible to obtain better performance by increasing the SCR of the data. Targets with higher SCR can be obtained by improving the azimuth resolution. We processed the single-look-complex images with 0.6- $\mathrm{m}$ resolution in azimuth $(900 \mathrm{~m}$ of synthetic aperture in midrange). The ARMA filter length is $90 \mathrm{~m}$. Improvement in the convergence behavior is noticeable when using the filter, leading to more accurate results.

Fig. 5 shows the estimation results from WPCA. The graphic in Fig. 5(a) and (b) are the horizontal and vertical components of the motion deviations of the master image, respectively. Fig. 5(c) and (d) shows the same for the slave image. The convergence is achieved at the second iteration for the master and at the fourth iteration for the slave, with about $0.5-\mathrm{cm}$ $(0.25 \mathrm{rad})$ accuracy. That corresponds to about three times the theoretical accuracy computed [(29)] for this data set $\left(\beta_{\phi}=3\right)$. Higher residual errors were found in the slave image.

To show the improvement in the focusing of the image, Fig. 6 shows the IRF of a CR of the slave image before and after the application of WPCA. The analyzed corner is located at the top of the image (inside of a white circle) in Fig. 4. For the master image, no significant improvement in the focusing is observed, since the estimated errors are small. It is clear to see that, after WPCA, the focusing of the IRF of the slave, which has significant residual errors, has improved, leading to the corrections of the IRF positioning and phase. These corrections contribute for a better interferogram generation.

Since the main objective when compensating for residual motion errors is the generation of accurate interferograms, free of misregistration and phase errors, we focus now on the interferometric data. The interferometric data were coregistered according only to the nominal baseline. Fig. 7 shows the improvement in the coherence through the use of WPCA. Fig. 8 shows the residual interferograms, i.e., the topographic-phase component (from a DEM) is removed from the interferograms, before and after residual motion compensation. Fig. 8(a) shows the residual interferogram before WPCA, and Fig. 8(c) shows it after applying WPCA. There is a clear improvement in the phase accuracy when using the WPCA.

The corrected interferogram still contains the phase due to the unknown global offset and linear components not 


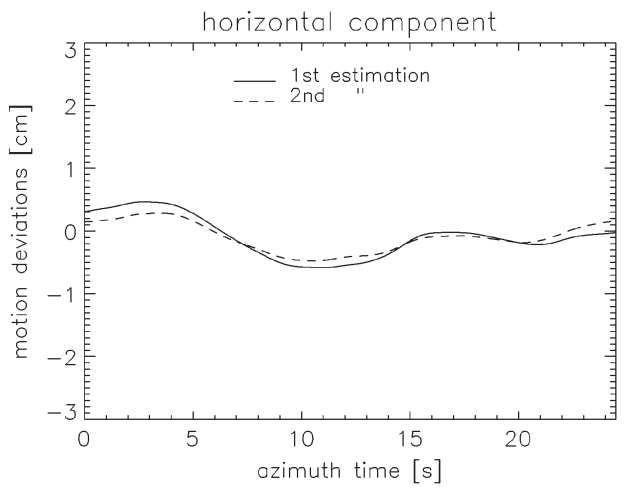

(a) master

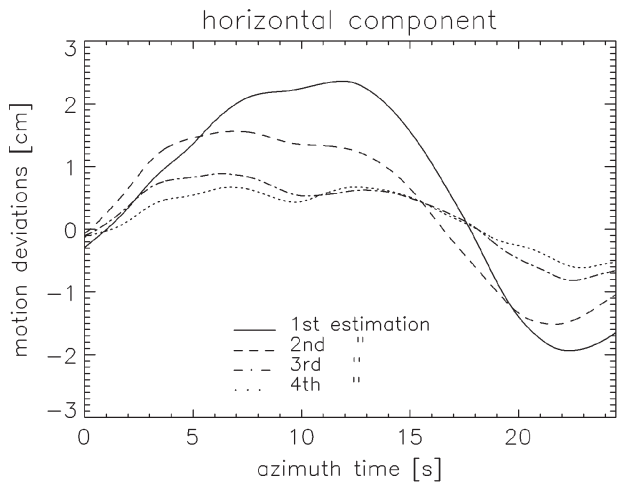

(c) slave $(B=6.44 \mathrm{~m})$

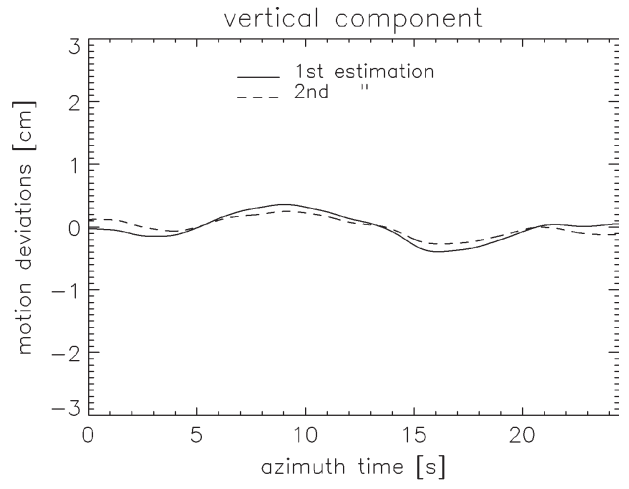

(b) master

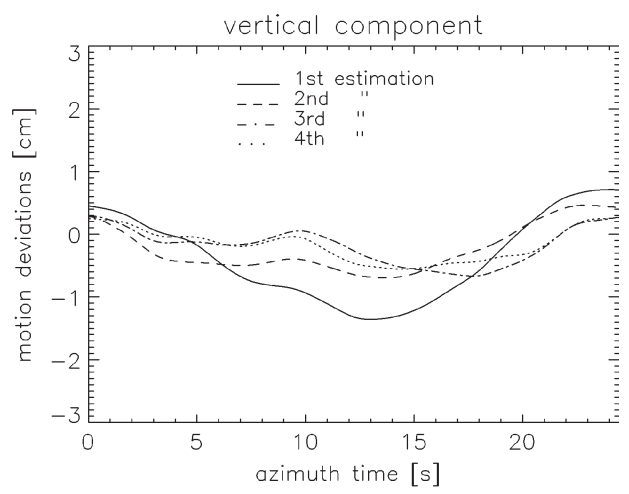

(d) slave $(B=6.44 \mathrm{~m})$

Fig. 5. Residual-motion estimations for the master image. (a) Horizontal deviations. (b) Vertical deviations. For the slave image. (c) Horizontal deviations. (d) Vertical deviations.

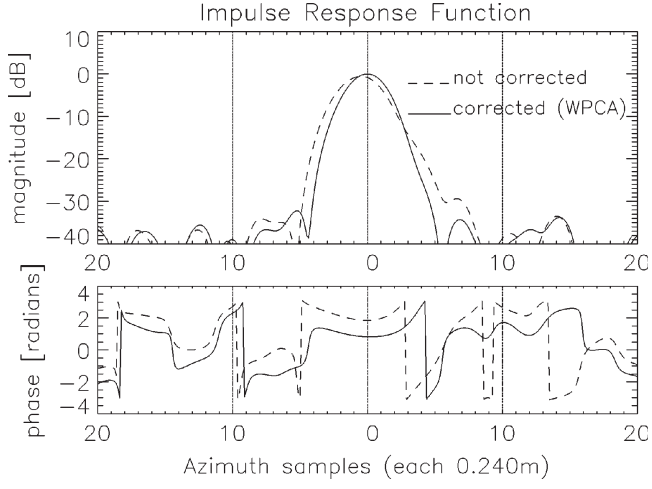

Fig. 6. IRF of the CR, located in the bottom of the amplitude image (slave) in a circle (see Fig. 4).

estimated because of the double-derivative formulation. To remove the global components, we fit the residual interferogram into a linear model described in (31). This could be also done with few CRs strategically placed. To have a fair idea of the improvement, we removed also the global offset and linear component from the interferogram with motion errors. Fig. 8(b) shows the interferogram after global terms removal but without WPCA, and Fig. 8(d) shows the final residual interferogram, i.e., after WPCA and global terms removal. The final corrected interferogram contains mainly phase artifacts from DEM errors.

\section{B. Comparison With Multisquint}

Next, a comparison with the residual-motion-error estimations obtained with WPCA and multisquint is performed. The

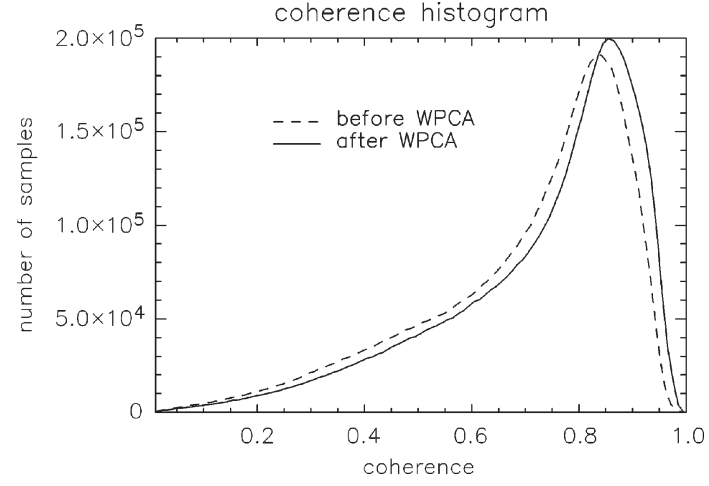

Fig. 7. Histogram of the coherence (a) before and (b) after WPCA.

objective is to evaluate if WPCA achieves the same quality interferograms as multisquint with high-coherence data. The data used is the one presented in Section IV-A. The sublooks center-frequency separation in multisquint is $20 \mathrm{~Hz}$, and eight overlapping looks were used.

Fig. 9 shows the multisquint estimation for the motion errors. Since multisquint is an interferometric approach, the estimated motion is the relative one between master and slave, i.e., the residual baseline variation. As observed, multisquint works properly, since it achieves fast convergence.

Fig. 8(e) and (f) shows the residual interferograms obtained with multisquint. The quality of the residual interferograms obtained by multsquint and WPCA are similar. Some differences can be observed in the top part of the interferogram at far range.

Fig. 10 shows the comparison between the WPCA and multisquint estimated relative motion error (master to slave). 


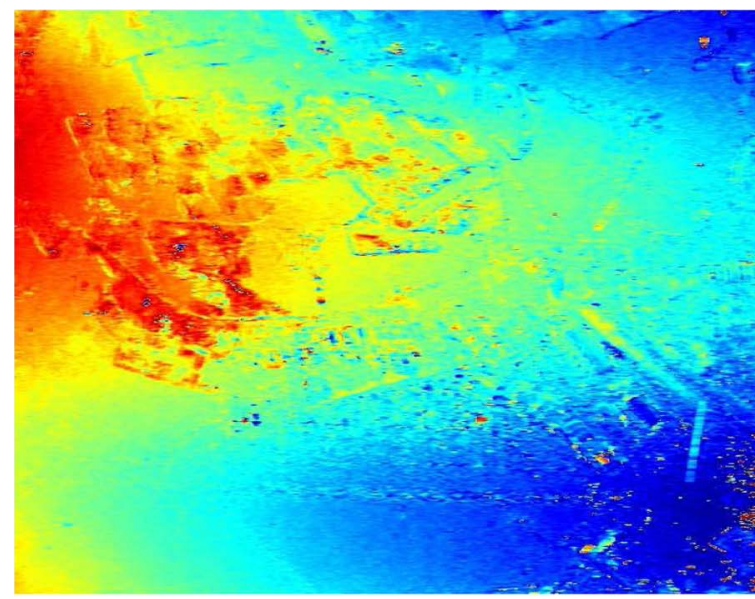

(a) before correction

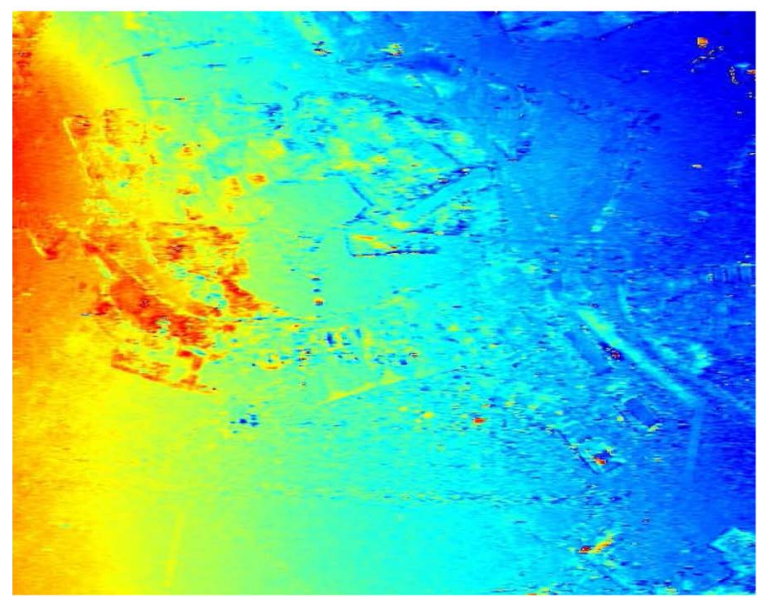

(c) after WPCA

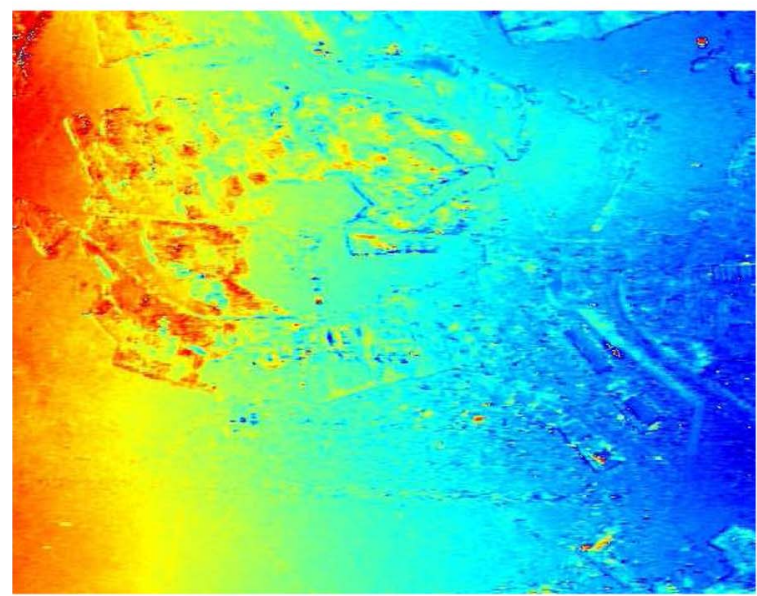

(e) after multisquint

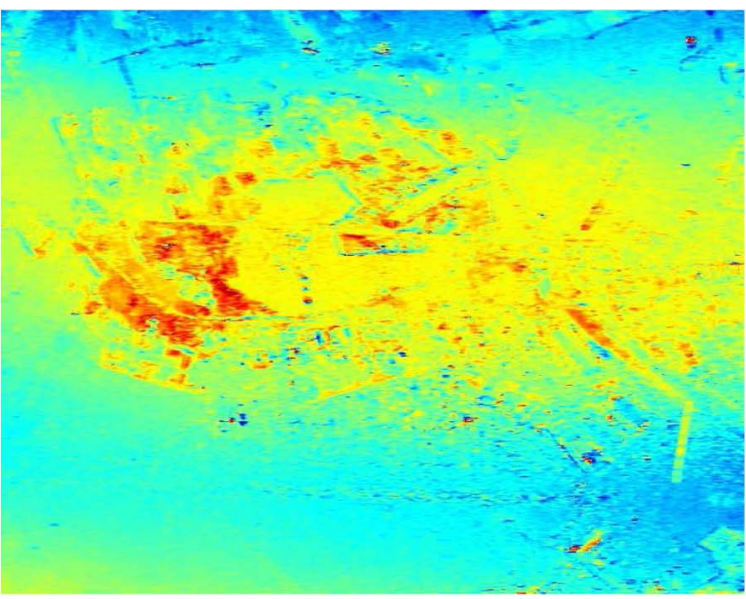

(b) before correction (global terms removed)

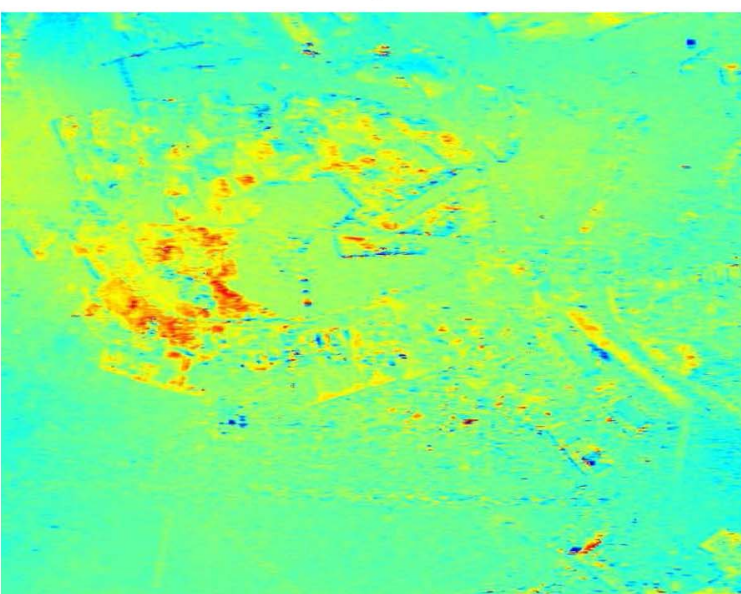

(d) after WPCA (global terms removed)

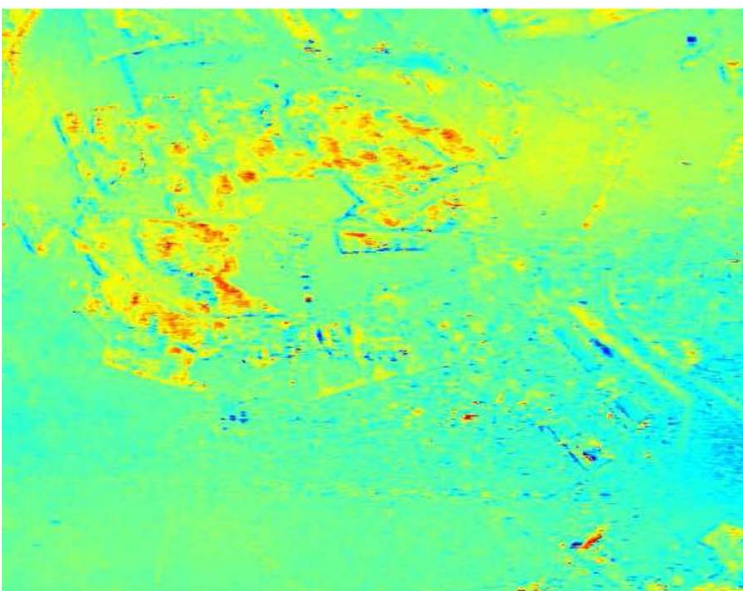

(f) after multisquint (global terms removed)

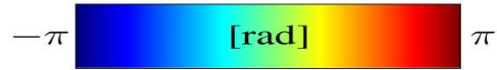

Fig. 8. Residual interferograms (left) without and (right) with removal of constant and linear global phase terms. Residual interferograms (a) and (b) before residual-motion-error compensation, (c) and (d) after WPCA, and (e) and (f) after multisquint $(B=6.44 \mathrm{~m})$.

In order to compare them, only the total estimation needs to be taken into account. This is because the total motion-error compensation performed in the images corresponds to the sum of all estimations at different iterations. Therefore, the estimations at different iterations are added up, leading to the total motion estimation for each method. After the total computation, the WPCA total motion estimation of the master is subtracted from the slave total estimation to have the variable 


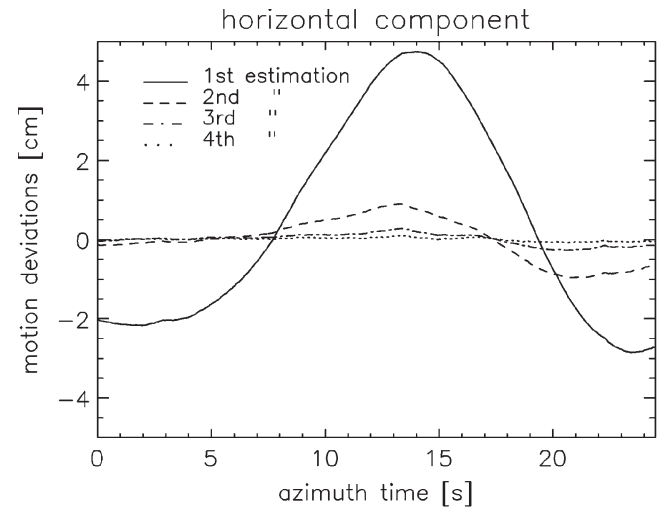

(a)

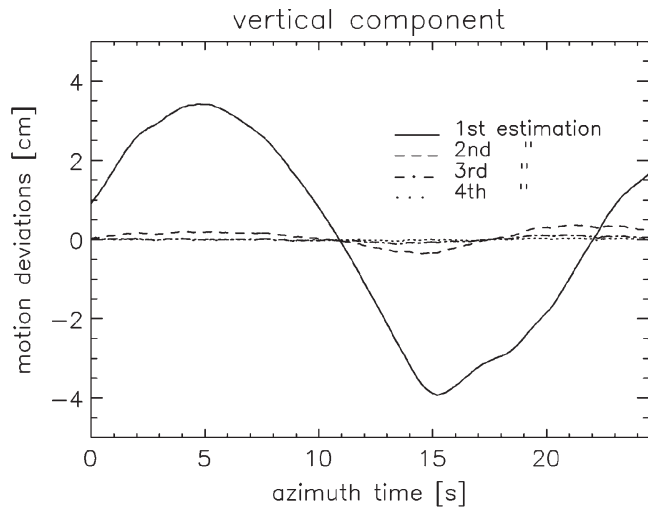

(b)

Fig. 9. Multisquint estimations of the (a) horizontal and (b) vertical component of the residual variable baseline (motion deviations relative to master).

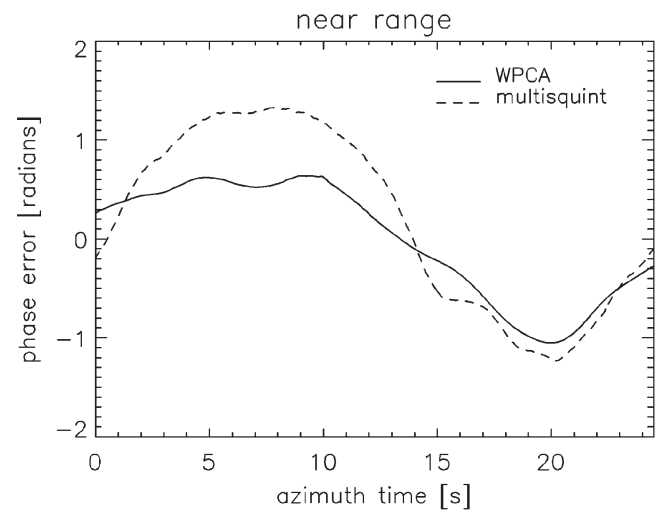

(a)

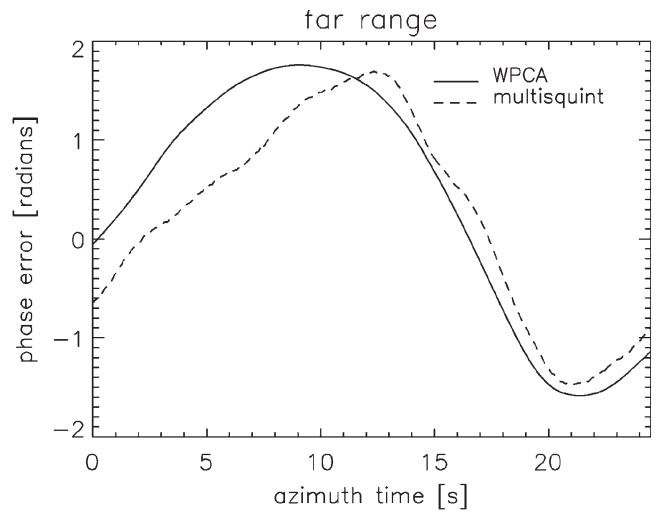

(b)

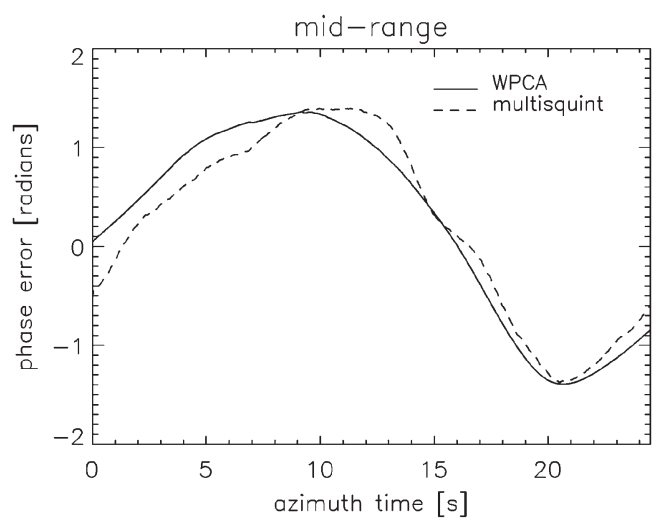

(c)

Fig. 10. Comparison of the WPCA and multisquint relative phase-error estimations between master and slave at (a) near range, (b) far range, and (c) midrange.

residual-baseline-estimation equivalent to the one delivered by multisquint. Since the inversion of the range-dependent model does not have a unique solution, the comparison of the estimations cannot be done with the horizontal and vertical components directly. A comparison is possible by evaluating the estimated phase error in line-of-sight, which is the one that is effectively being used to compensate for the motion deviations. Thus, the estimated phase errors are evaluated in near, mid, and far ranges to allow a fair comparison over the whole data take.

The differences between WPCA and multisquint estimations are mainly located in the beginning of the data take, as also noticeable in the interferograms (top of the image). In near and far ranges, the errors are clearly larger than in midrange. This is attributed to the range-dependent model. A conclusion about which of the algorithms performs better is difficult due to the totally different nature of the algorithms, and further work is necessary. Nevertheless, we can conclude that WPCA is able to obtain a performance comparable to multisquint.

\section{CONCLUSION}

In this paper, an autofocus approach for repeat-pass SAR interferometry, where the curvature of the residual motion error of each image is estimated and compensated independently, is proposed. It involves the use of a new and robust autofocus 
algorithm, the WPCA. The proposed approach leads to a better focused image, correct nominal IRF positioning and phase. As a consequence, better coherence and interferometric phase free of residual motion errors can be achieved with nominal coregistration. The WPCA algorithm is able to estimate residual phase errors with an accuracy under 0.25 rad at L-band, satisfying the requirements for typical airborne D-InSAR applications. Tests to evaluate the performance of the WPCA for different test sites and different radar wavelengths have been executed and are shown in [36].

As long the residual-motion estimations are converging, the WPCA algorithm is working properly. Underestimation may occur if the ARMA filter length is too large (practically, 90-m length causes no underestimation at all) and/or the processed synthetic aperture is not large enough as it is the case for E-SAR data at $\mathrm{C}$ - or X-band. Tests in $\mathrm{C}$-band with an aperture of $250 \mathrm{~m}$ (0.6-m resolution) have shown some underestimation problems, although noticeable mitigation of residual errors was also observed. Due to the smaller synthetic aperture of stripmap images at higher frequencies, such as C- and $\mathrm{X}$-band, the residual motion errors within the synthetic aperture becomes practically a constant or a linear function. In this case, WPCA is not sensible enough to estimate residual motion errors accurately, leading to underestimated results. To overcome this problem, images at $\mathrm{C}$ - or $\mathrm{X}$-band have to be acquired and processed with very high resolution $(\leq 0.2 \mathrm{~cm})$ to allow a larger synthetic aperture and, therefore, higher motion-acceleration signal power. For a given sensor, exact evaluation is required, considering the motion spectrum of the airborne platform.

Repeat-pass interferometry based on WPCA gains in robustness and reliability, since its performance does not deteriorate with temporal decorrelation. This opens the possibility to perform robust airborne D-InSAR for low-coherence and longterm interferograms at any frequency band. Additionally, no assumption is needed to be made on the interferometric phase related to the along-track terrain displacements in order to estimate the motion errors. It is important to note that optimal performance of phase-error-estimation algorithms is achieved only when using topography- and aperture-dependent MoComp. In the case of repeat-pass interferometry, this becomes mandatory.

We may say that the multisquint relies on the interferometric coherence, while WPCA relies on high SCR. Depending on the scene under consideration and the interferometric application, one approach can be more appropriate than the other. For the test site used in this paper, mostly vegetated (L-band, processed with 0.6-m azimuth resolution), the achieved estimation accuracy was about three $\left(\beta_{\phi}=3\right)$ times greater than the theoretical one.

Once the differential interferograms are free of residual errors, advanced interferometry techniques like permanent scatterers or small baseline can be successfully applied [37]-[41]. Further work consists in applying the autofocus approach (WPCA) for real repeat-pass applications to measure terrain deformations (D-InSAR) with airborne data.

\section{ACKNOWLEDGMENT}

The authors would like to thank M. Catillo for the initial investigations on PGA algorithms and the anonymous reviewers for the helpful comments and suggestions.

\section{REFERENCES}

[1] J. C. Kirk, "Motion compensation for synthetic aperture radar," IEEE Trans. Aerosp. Electron. Syst., vol. AES-11, no. 3, pp. 338-348, May 1975.

[2] D. Blacknell, A. Freeman, S. Quegan, I. A. Ward, I. Finley, C. J. Oliver, R. G. White, and J. Wood, "Geometric accuracy in airborne SAR images," IEEE Trans. Aerosp. Electron. Syst., vol. 25, no. 2, pp. 241-258, Mar. 1989.

[3] S. Buckreuss, "Motion errors in an airborne synthetic aperture radar system," Eur. Trans. Telecommun., vol. 2, no. 6, pp. 655-664, Nov./Dec. 1991.

[4] C. E. Mancill and J. M. Swiger, "A map drift autofocus technique for correcting higher order SAR phase errors," in Proc. 27th Annu. Tri-Service Radar Symp. Rec., Monterey, CA, Jun. 1981, pp. 523-525.

[5] P. H. Eichel and C. V. Jakowatz, Jr., "Phase-gradient algorithm as an optimal estimator of the phase derivative," Opt. Lett., vol. 14, no. 20, pp. 1101-1103, Oct. 1989.

[6] J. R. Moreira, "A new method of aircraft motion error extraction from radar raw data for real time motion compensation," IEEE Trans. Geosci. Remote Sens., vol. 28, no. 4, pp. 620-626, Jul. 1990.

[7] C. V. Jakowatz, Jr. and D. E. Whal, "Eigenvector method for maximumlikelihood estimation of phase errors in synthetic-aperture radar imagery," J. Opt. Soc. Amer. A, Opt. Image Sci., vol. 10, no. 12, pp. 2539-2546, Oct. 1993.

[8] D. E. Wahl, P. Eichel, D. C. Ghiglia, and C. V. Jakowatz, Jr., "Phase gradient autofocus-A robust tool for high resolution SAR phase correction,' IEEE Trans. Aerosp. Electron. Syst., vol. 30, no. 3, pp. 827-835, Jul. 1994.

[9] A. Moreira and Y. Huang, "Airborne SAR processing of highly squinted data using a chirp scaling approach with integrated motion compensation," IEEE Trans. Geosci. Remote Sens., vol. 32, no. 5, pp. 1029-1040, Sep. 1994.

[10] R. Scheiber, A. Reigber, A. Ulbricht, K. P. Papathanassiou, R. Horn, S. Buckreuß, and A. Moreira, "Overview of interferometric data acquisition and processing modes of the experimental airborne SAR system of DLR," in Proc. IGARSS, Hamburg, Germany, Jun. 1999, pp. 35-37.

[11] S. Hensley, S. Rosen, S. Madsen, and B. Chapman, "Some airborne repeat-pass deformation measurements using the AIRSAR," in Proc. EUSAR, Dresden, Germany, May 2006.

[12] G. Fornaro, E. Lanari, E. Sansosti, G. Franceschetti, S. Perna, A. Gois, and J. Moreira, "Airborne differential interferometry: X-band experiments," in Proc. IGARSS, Anchorage, AK, Sep. 2004, pp. 3329-3332.

[13] H. Cantalloube and P. Dubois-Fernandez, "Airborne X-band SAR imaging with $10 \mathrm{~cm}$ resolution: Technical challenge and preliminary results," Proc. Inst. Electr. Eng.-Radar Sonar Navig., vol. 153, no. 2, pp. 163-176, Apr. 2006.

[14] D. R. Stevens, I. G. Cumming, and A. L. Gray, "Options for airborne interferometric SAR motion compensation," IEEE Trans. Geosci. Remote Sens., vol. 33, no. 2, pp. 409-420, Mar. 1995.

[15] A. Reigber, "Correction of residual motion errors in airborne SAR interferometry," Electron. Lett., vol. 37, no. 17, pp. 1083-1084, Aug. 2001.

[16] P. Prats and J. J. Mallorqui, "Estimation of azimuth phase undulations with multisquint processing in airborne interferometric SAR images," IEEE Trans. Geosci. Remote Sens., vol. 41, no. 6, pp. 1530-1533, Jun. 2003.

[17] K. A. C. de Macedo, C. Andres, and R. Scheiber, "On the requirements of SAR processing for airborne differential interferometry," in Proc. IGARSS, Seoul, Korea, Jul. 2005, pp. 2693-2696.

[18] G. Fornaro, G. Franceschetti, and S. Perna, "Motion compensation errors: Effects on the accuracy of airborne SAR images," IEEE Trans. Aerosp. Electron. Syst., vol. 41, no. 4, pp. 1338-1352, Oct. 2005.

[19] R. Scheiber and A. Moreira, "Coregistration of interferometric SAR images using spectral diversity," IEEE Trans. Geosci. Remote Sens., vol. 38, no. 5, pp. 2179-2191, Sep. 2000.

[20] H. L. Chan and T. S. Yeo, "Noniterative quality phase-gradient autofocus (QPGA) algorithm for spotlight SAR imagery," IEEE Trans. Geosci. Remote Sens., vol. 36, no. 5, pp. 1531-1539, Sep. 1998.

[21] W. Ye, T. S. Yeo, and Z. Bao, "Weighted least-squares estimation of phase errors for SAR/ISAR autofocus," IEEE Trans. Geosci. Remote Sens., vol. 37, no. 5, pp. 2487-2494, Sep. 1999.

[22] W. G. Carrara, R. S. Goodman, and R. M. Majewski, Spotlight Synthetic Aperture Radar-Signal Processing Algorithms. Boston, MA: Artech House, 1995.

[23] D. E. Wahl, C. V. Jakowatz, Jr., P. A. Thompson, and D. C. Ghiglia, "New approach to strip-map SAR autofocus," in Proc. 6th IEEE Digital Signal Process. Workshop, Yosemite National Park, CA, Oct. 1994, pp. 53-56.

[24] P. Prats, C. Andres, R. Scheiber, K. A. C. de Macedo, J. Fischer, and A. Reigber, "Glacier displacement field estimation using airborne SAR interferometry," in Proc. IGARSS, Jul. 2007, pp. 2098-2101. 
[25] S. M. Kay, Fundamentals of Statistical Signal Processing: Estimation Theory. Upper Saddle River, NJ: Prentice-Hall, 1993.

[26] D. G. Thompson, J. S. Bates, D. V. Arnold, and D. G. Long, "Extending the phase gradient autofocus algorithm for low-altitude stripmap mode SAR," in Proc. IGARSS, Hamburg, Germany, Jul. 1999, pp. 564-566.

[27] J. H. Callow, "Signal processing for synthetic aperture sonar image enhancement," Ph.D. dissertation, Dept. Elect. Electron. Eng., Univ. Canterbury, Christchurch, New Zealand, 2003.

[28] T. J. Sutton, S. A. Chapman, and H. D. Griffiths, "Robustness and effectiveness of autofocus algorithms applied to diverse seabed environments," in Proc. 5th ECUA, Lyon, France, Jul. 2000, pp. 407-412.

[29] P. T. Gough, M. P. Hayes, and D. R. Wilkinson, "Strip-map path correction using phase matching autofocus," in Proc. 5th ECUA, Lyon, France, Jul. 2000, pp. 412-418.

[30] M. P. Hayes, J. H. Callow, and P. T. Gough, "Strip-map phase gradient autofocus," in Proc. Image Vis. Comput. New Zealand, Auckland, New Zealand, Dec. 2002, pp. 71-76.

[31] S. N. Madsen, "Estimating the Doppler centroid of SAR data," IEEE Trans. Aerosp. Electron. Syst., vol. 25, no. 2, pp. 134-140, Mar. 1989.

[32] P. Prats, A. Reigber, and J. J. Mallorqui, "Interpolation-free coregistration and phase-correction of airborne SAR interferograms," IEEE Geosci. Remote Sens. Lett., vol. 1, no. 3, pp. 188-191, Jul. 2004.

[33] A. Reigber, P. Prats, and J. J. Mallorqui, "Refined estimation of timevarying baseline errors in airborne SAR interferometry," IEEE Geosci. Remote Sens. Lett., vol. 3, no. 1, pp. 145-149, Jan. 2006.

[34] K. A. C. de Macedo and R. Scheiber, "Precise topography- and aperturedependent motion compensation for airborne SAR," IEEE Geosci. Remote Sens. Lett., vol. 2, no. 2, pp. 172-176, Apr. 2005.

[35] P. Prats, A. Reigber, and J. J. Mallorqui, "Topography-dependent motion compensation for repeat-pass interferometric SAR systems," IEEE Geosci. Remote Sens. Lett., vol. 2, no. 2, pp. 206-210, Apr. 2005.

[36] K. A. C. de Macedo, "New processing methodology for airborne repeatpass SAR interferometry," Ph.D. dissertation, Dept. Elect. Eng. Inf. Technol., Univ. Karlsruhe, Karlsruhe, Germany, 2007.

[37] A. Ferretti, C. Prati, and F. Rocca, "Permanent scatterers in SAR interferometry," IEEE Trans. Geosci. Remote Sens., vol. 39, no. 1, pp. 8-20, Jan. 2001

[38] A. Ferretti, G. Savio, R. Barzaghi, A. Borghi, S. Musazzi, F. Novali, C. Prati, and F. Rocca, "Submillimeter accuracy of InSAR time series: Experimental validation," IEEE Trans. Geosci. Remote Sens., vol. 45, no. 5, pp. 1142-1153, May 2007.

[39] P. Berardino, G. Fornaro, R. Lanari, and E. Sansosti, "A new algorithm for surface deformation monitoring based on small baseline differential SAR interferograms," IEEE Trans. Geosci. Remote Sens., vol. 40, no. 11, pp. 2375-2383, Nov. 2002.

[40] O. Mora, J. J. Mallorqui, and A. Broquetas, "Linear and nonlinear terrain deformation maps from a reduced set of interferometric SAR images," IEEE Trans. Geosci. Remote Sens., vol. 41, no. 10, pp. 2243-2253, Oct. 2003.

[41] P. Prats, A. Reigber, J. J. Mallorqui, R. Scheiber, and A. Moreira, "Estimation of the temporal evolution of the deformation using airborne differential SAR interferometry," IEEE Trans. Geosci. Remote Sens., vol. 46, no. 4, pp. 1065-1078, Apr. 2008.

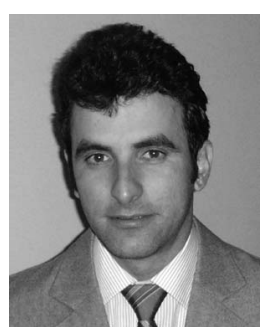

Karlus A. Câmara de Macedo (M'08) was born in Natal, Brazil, in 1974. He received the Diploma degree in electrical engineering from the University of Brasília, Brasília, Brazil, in 1999, the M.Sc. degree from the Institute of Aeronautical Technology (ITA), São José dos Campos, Brazil, in 2001, with research work on SAR geocoding, and the Ph.D. degree in electrical engineering from the University of Karlsruhe, Karlsruhe, Germany, in 2008, with a thesis on airborne differential SAR interferometry.

From 2000 to 2002, he researched on SAR in the frame of the CCSIVAM and CNS/ATM project at ITA, Brazil. From 2003 to 2007, he was with the Microwaves and Radar Institute, German Aerospace Center (DLR), Oberpfaffenhofen, Germany, doing research on D-InSAR with the E-SAR system of DLR. Between 2004 and 2005, he joined the cooperation work on airborne D-InSAR between DLR, the Universitat Politècnica de Catalunya (Barcelona, Spain), and the Technische Universität Berlin (Berlin, Germany). Since February 2008, he has been with Orbisat Remote Sensing, São José dos Campos, Brazil, working in the field of SAR technology. His current research interests include airborne repeat-pass SAR interferometry, D-InSAR applications, and new SAR system design.

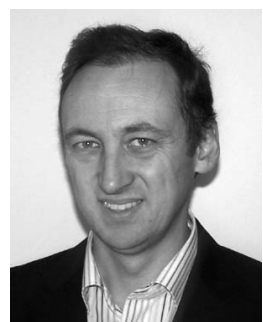

Rolf Scheiber received the Diploma degree in electrical engineering from the Technical University of Munich, Munich, Germany, in 1994 and the Ph.D. degree in electrical engineering with a thesis on airborne SAR interferometry from the University of Karlsruhe, Karlsruhe, Germany, in 2003.

Since 1994, he has been with the Microwaves and Radar Institute, German Aerospace Center (DLR), Oberpfaffenhofen, where he developed the operational high-precision interferometric SAR processor for its E-SAR airborne sensor, and since 2001, he has been heading the SAR Signal Processing Group, SAR Technology Department, where he is currently responsible for the E-SAR campaign data processing as well as the development of the processing software for the new airborne sensor F-SAR. His current research interests include algorithm development for highresolution air- and spaceborne SAR focusing, SAR interferometry, differential SAR interferometry, SAR tomography, as well as radar subsurface-sounding algorithms and applications.

Dr. Scheiber was the recipient of the GRSS Transactions Prize Paper Award in 1997 as coauthor of the contribution "Extended Chirp Scaling Algorithm for Air- and Spaceborne SAR Data Processing in Stripmap and ScanSAR Imaging Modes."

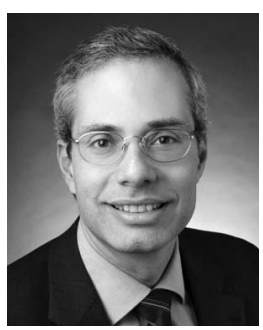

Alberto Moreira (M'92-SM'96-F'04) was born in São José dos Campos, Brazil, in 1962. He received the B.S.E.E. and M.S.E.E. degrees from the Aeronautical Technological Institute, Brazil, in 1984 and 1986, respectively, and the Eng.Dr. degree (with honors) from the Technical University of Munich, Munich, Germany, in 1993.

In 2003, he received a full professorship from the University of Karlsruhe, Karlsruhe, Germany, in the field of Microwave Remote Sensing. From 1996 to 2001, he was a Chief Scientist and Engineer with the SAR Technology Department, Microwaves and Radar Institute, German Aerospace Center (DLR), Oberpfaffenhofen, where he has been the Director since 2001. Under his leadership, the DLR airborne SAR system, E-SAR, has been upgraded to operate in innovative imaging modes like polarimetric SAR interferometry and SAR tomography. The institute contributes to several scientific programs and space projects for actual and future air- and spaceborne SAR missions like TerraSAR-X and Sentinel-1. In 2006, the mission proposal TanDEM-X led by his institute has been approved for the realization phase, where he is the Principal Investigator. His professional interests and research areas encompass radar end-to-end system design and analysis, innovative microwave techniques and system concepts, signal processing, and remotesensing applications.

Prof. Moreira is serving as a member of the IEEE GRSS Administrative Committee (1999-2001, 2004-2007, 2008-2009 as Executive Vice President). He was also the Founder and Chair of the German Chapter of the GRSS (2003-2008). He was an Associate Editor for the IEEE GEOscIENCE AND REMOTE SENSING LetTERS (2003-2007) and is currently serving as Associate Editor for the IEEE TRANSACTIONS ON GEOSCIENCE AND REMOTE SENSING. Since 2003, he has also been serving as a member of the Board of Directors of the Information Technology Society of VDE (German Association for Electrical, Electronic, and Information Technologies). He was the recipient of the DLR Science Award in 1995. He and his colleagues were the recipient of the GRSS Transactions Prize Paper Awards in 1997 and 2001. He was also the recipient of the IEEE Nathanson Award in 1999 and the IEEE Kiyo Tomiyasu Award in 2007. He has been contributing to the successful series of the European SAR conferences, since 1996, as member of the Technical Program Committee, Technical Chairman in 2000, Awards Chairman from 2002 to 2004, General Chairman in 2006, and Cochairman in 2008. 